\title{
Current rectification in molecular junctions produced by local potential fields
}

\author{
Tomasz Kostyrko \\ Faculty of Physics, A. Mickiewicz University, ul. Umultowska 85, 61-614 Poznań, Poland \\ Víctor M. García-Suárez \\ Departamento de Física, Universidad de Oviedo \& CINN, 33007 Oviedo, Spain \\ and Department of Physics, Lancaster University, Lancaster LA1 4YB, United Kingdom \\ Colin J. Lambert \\ Department of Physics, Lancaster University, Lancaster LA1 4YB, United Kingdom
}

Bogdan R. Bułka

Institute of Molecular Physics, Polish Academy of Science, Smoluchowskiego 17, 60-179 Poznań, Poland

(Received 10 November 2009; revised manuscript received 13 December 2009; published 5 February 2010)

\begin{abstract}
The transport properties of an octane-dithiol (ODT) molecule coupled to $\mathrm{Au}(001)$ leads are analyzed using density-functional theory (DFT) and nonequilibrium Green's functions. It is shown that a symmetric molecule can turn into a diode under influence of a local electric field created by an external charged probe. The origin of the asymmetry of the current-voltage $(I-V)$ dependence is traced back to the appearance of a probe-induced quasilocal state in the pseudogap of the ODT molecule. The induced state affects electron transport provided it is close to the Fermi level of the leads. An asymmetric placement of the charged probe along the alkane chain makes the induced quasilocal state in the energy gap very sensitive to the bias voltage and results in rectification of the current. The results based on DFT are supported by independent calculations using a simple one-particle model Hamiltonian.
\end{abstract}

DOI: 10.1103/PhysRevB.81.085308

PACS number(s): 73.63.-b, 85.65.+h, 73.40.-c

\section{INTRODUCTION}

The quest for molecular diodes, which was started by Aviram and Ratner in 1974 (Ref. 1), has stimulated a number of experimental and theoretical investigations of molecular rectifiers. $^{2-16}$ The Aviram-Ratner mechanism is based on donor-insulator-acceptor molecules, where electrons are transferred inelastically from the acceptor to the donor for a certain bias value and polarity. It is also possible to have rectifying behaviors in case of coherent transport when asymmetries are present in the molecular structure. Such asymmetries can be produced, for instance, by asymmetric molecules or different contacts to the electrodes. The current starts to increase for a certain bias and polarity whenever molecular orbitals, which are located asymmetrically with respect to the leads, coincide with the Fermi level of one of the electrodes ${ }^{4,5,7}$ or when the energies of molecular orbitals localized on different parts of the molecule match inside the bias window. For the reverse polarity the orbitals located asymmetrically reach one of the Fermi levels at higher voltages in the first case and they separate in energy and do not match inside the bias window in the second case. The coherent mechanism gives rise to moderately high rectification ratios, $R R(V)=|I(V) / I(-V)|[$ where $I(V)$ denotes the current as a function of the voltage $V] .^{3}$ The theoretical calculations based on either coherent or incoherent mechanisms predict clear rectification behaviors but from a practical point of view success has been modest. ${ }^{2,17,18}$ Estimates using both parametric models and $a b$ initio studies have raised doubts about whether the molecular asymmetries could lead to a sizable $R R$ based on elastic scattering. ${ }^{5-8,11,12,14,15}$ Most of these studies report values of $R R$ which do not exceed a dozen or so. So far, however, the question whether there is an upper limit for the rectification value for a molecular diode, and what this value might be, did not receive an unambiguous answer.

The experimentally obtained values of $R R$ for molecular diodes are usually much less than 100 and are orders of magnitude smaller than the corresponding values in typical inorganic devices. ${ }^{9,10,16}$ Although much bigger values $\left(R R \sim 10^{3}\right)$ of $R R$ may be obtained after exposure of the self-assembled monolayers to air ${ }^{19}$ or by protonation, ${ }^{20}$ it is not clear the possible impact of the ionic conductivity on the current-voltage characteristics of the samples. $^{21}$

In order to design the best molecular diode it is necessary to optimize the main parameters governing the rectifying behavior of the device: highest occupied molecular orbital (HOMO)-lowest unoccupied molecular orbital (LUMO) energy gap, length of the intervening molecular bridge, the position of the HOMO-LUMO system with respect to the Fermi level of the leads, and the coupling strength of the molecular levels to the metallic levels of the leads. In both experimental and theoretical works this goal is commonly realized by a more or less arbitrary choice of molecular components, i.e., the donor and acceptor subunits and the connecting bridge.

In this paper, as an alternative approach to other molecular-scale rectification mechanisms, we carry out a systematic simulation study of rectification induced by the presence of a local external charge in the vicinity of a molecular bridge. In part, our study is motivated by related experiments involving carbon nanotubes with charged AFM tips acting as the source of a local potential. ${ }^{22}$ At a molecular scale such a 


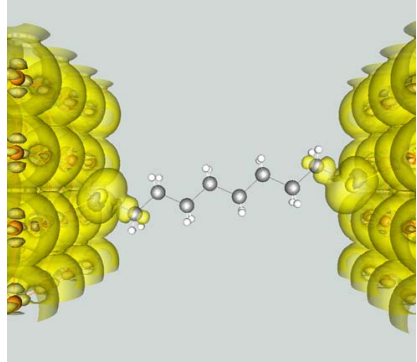

(a)

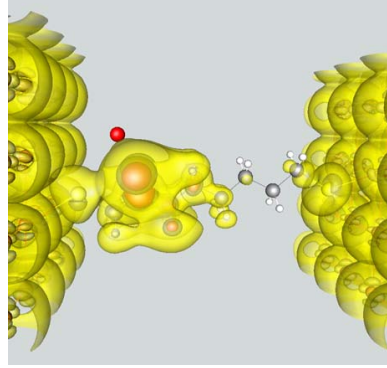

(b)
FIG. 1. (Color online) The ball-and-stick model of the junction with the ODT molecule and fragments of the gold leads used in our DFT computations. The regular balls indicate the positions of the atoms. The irregular contours in the figure show isosurfaces of LDOS (see text in the end of Sec. III for a discussion) near the energy $E_{\mathrm{F}}+1.2 \mathrm{eV}$ integrated in the energy window of width $1 \mathrm{eV}$. The left figure is for the junction without the probe and the right one is for the junction with the probe in position $\mathrm{C} 1$. The probe is shown with an additional (red) ball in the right figure.

symmetry-breaking charge may be located in the surrounding solvent or in a self-assembled monolayer matrix of shorter molecules with a substantial dipole moment. For our calculations we use an octane-dithiol (ODT) molecule coupled to gold leads, a system well studied in recent works. ${ }^{23}$

\section{SYSTEM SETUP AND PARAMETERS OF THE DFT COMPUTATIONS}

We model the molecular junction within the SMEAGOL methodology, ${ }^{24}$ where the system is divided into an extended molecule (formed by the molecule and some surface layers of gold atoms) and the periodic semi-infinite leads. In our case the extended molecule is the ODT molecule and $4 \times 4 \times 4$ and $4 \times 4 \times 5$ atomic sections of $\mathrm{Au}$ fcc lattice on the left and right sides of the junction, respectively (see Fig. 1). Periodic boundary conditions are applied in the directions parallel to the planes, i.e., transverse to the conduction direction. The solutions at the right and left boundaries of the extended molecule are matched to the solution obtained from an independent computation of the periodic leads. The total number of atoms in the unit cell was 170 . The spatial structure of the junction was defined in several stages. First, the structure of the periodic leads and the ODT molecule was independently optimized using a conjugate gradient method from SIESTA, ${ }^{25}$ with a maximum force tolerance of $0.01 \mathrm{eV} / \AA$. Next, the ODT molecule was attached in the hollow site position of the $\mathrm{Au}(001)$ surface of the leads and the molecular structure was optimized again (we did not allow at this stage for the relaxation of Au atoms) with the same parameters. In the course of the relaxation the bonding $\mathrm{S}$ atoms on both sides moved significantly to the bridge position of the $\mathrm{Au}(001)$ surface plane with dominating 2-Au coordination.

In the SIESTA computations ${ }^{26}$ we used the local-density approximation with the Perdew and Zunger ${ }^{27}$ model for the exchange and correlation potential. The core electrons were
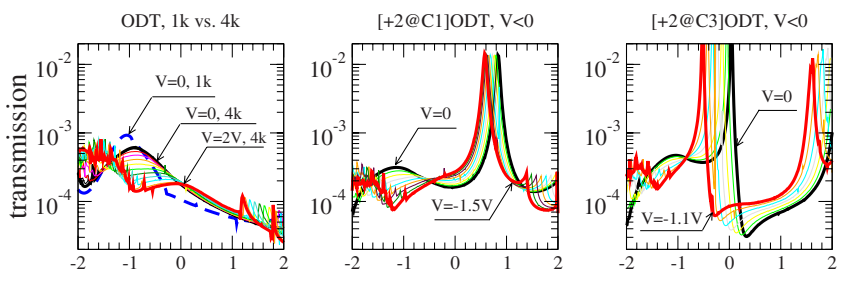

$[\mathrm{Q} @ \mathrm{C} 1] \mathrm{ODT}, \mathrm{V}=0$
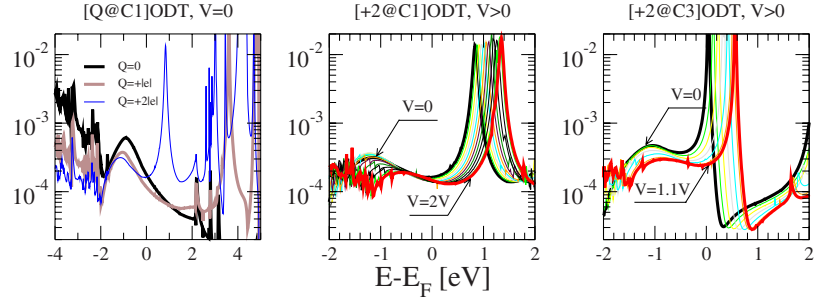

FIG. 2. (Color online) Evolution of the transmission versus energy near the Fermi level $\left(E_{\mathrm{F}} \equiv 0\right)$ as a function of the bias voltage for the different values and the positions of the probe charge obtained from SMEAGOL for the junction with ODT. All the plotted curves but one correspond to $4 k$ computations. Left column, upper row: the junction without the probe; $4 k$ results for $0<V<2 \mathrm{~V}$ are compared with $1 k$ for $V=0$. Left column, lower row: transmission function for various values of the charge probe in the extended energy scale. Middle column: the probe is positioned near the leftmost $\mathrm{C}$ atom (position $\mathrm{C} 1$, as in Fig. 1) of the ODT chain at a distance ca. $4 \AA$ from the carbon atom $(V<0$ : upper row; $V>0$ : lower row). Right column: the probe is shifted to the position of third $\mathrm{C}$ atom from the left, $\mathrm{C} 3$ ( $V<0$ : upper row; $V>0$ : lower row). The heavy lines show the results for the limiting values of voltage indicated with arrows and the thin ones are for intermediate values of voltage.

represented by pseudopotentials obtained using the Troullier and Martins method. ${ }^{28}$ For all atoms in the system we employed a single-zeta basis set. This restriction was necessary due to the memory limitations of our computation facilities especially in the subsequent SMEAGOL transport computations. However, tests performed with smaller gold leads and a double-zeta polarized basis set or the same gold leads and double-zeta or single-zeta polarized basis sets showed the same qualitative trends in the transport properties and the electronic structure [density of states (DOS)]. The radii of the pseudoatomic orbitals were fixed by an energy shift parameter of $0.02 \mathrm{Ry}$. The mesh cutoff parameter was set to 200 Ry and we used an additional grid cell sampling.

To simulate the effect of the charge probe we used an alkali atom (charge $+|e|$ ) or an alkaline-earth atom (charge $+2|e|$ ) with the basis restricted to just the $s$ orbital and a very small cutoff radius. This trick raises the atomic level of the probe atom far above the Fermi level of the leads completely depleting the $s$ orbital. In effect, the auxiliary atom can be considered to be a simple point charge, whose wave function has no overlap with the wave functions of ODT and gold. The unit cell remained exactly neutral in the SIESTA computation and was approximately neutral in the SMEAGOL computation. The atomic forces, upon introducing the probe atom, turned out to be quite large due to Coulomb attraction between the displaced electronic charges and the 
ionic charge of the probe center. However, we did not reoptimize the spatial structure of the unit cell at this stage, since the charge probe is meant to be an abstract representation of a local potential which could have various origins.

\section{RESULTS}

Figure 2 shows the computed electron transmission coefficients obtained for both $1 k$ point and $4 k$ points $^{26}$ in the transverse direction of the Brillouin zone. Since the large transverse size of the unit cell produces a large separation between different copies of the molecular junction, the results are a representative of transport through an individual molecule. Furthermore, a sufficiently large transverse unit cell needs only a small number of transverse $k$ points to approach the limit of the infinite surface. In our case, however, we can see that the difference between $1 k$ - and $4 k$-point calculations is still noticeable, although the main features of the results remain qualitatively the same. As shown in Fig. 2, a wide (ca. $2 \mathrm{eV}$ ) transmission peak is located at about $1 \mathrm{eV}$ below the Fermi level of the system. The peak is broad because it comes from molecular orbitals which are well coupled to the electronic states of the leads, i.e., the sulfur $3 \mathrm{~s}$ and especially $3 p$ orbitals. Note that the maximum height of the peak is much less than unity, because it arises from two almost energetically equivalent $s$ orbitals at opposite sites of the junction ${ }^{29,30}$ with an effective coupling, which decreases exponentially with the length of the molecular backbone. Under a bias voltage the peak splits into two peaks, with one moving upward and the other downward in energy. This behavior is due to the influence of the potential ramp of the applied voltage, which induces an energetic inequivalence of the sulfur orbitals at opposite ends of the molecule.

The influence of the charge of the probe on the zerovoltage transmission is presented in the lower left panel of Fig. 2. In the vicinity of the Fermi level the $Q=+|e|$ charge merely produces a rather uniform suppression of the wide transmission peak located about $1 \mathrm{eV}$ below $E_{\mathrm{F}}$. More important is the appearance of a rather prominent narrow transmission peak located at $E \sim 3.6 \mathrm{eV}$ above $E_{\mathrm{F}}$ and which does not have a counterpart in the transmission without the probe. For the $Q=+|e|$ charge probe the peak will not manifest itself in the corresponding $I-V$ dependence, because it is too far from the Fermi level and would not enter the source-drain voltage window for moderate $(|V|<2 \mathrm{~V})$ voltage values. However, increase in the charge of the probe to a value of $+2|e|$ appears to shift this peak to within a distance of $\sim 1 \mathrm{eV}$ from $E_{\mathrm{F}}$, which makes it important for the transport for $|V|<2 \mathrm{~V}$.

By comparing the middle and the right columns of Fig. 2 one can note a significant dependence of the transmission peak on the position of the charge probe. Shifting the probe from the left side (position $\mathrm{C} 1$ ) of the chain to a position closer to the center (C3) increases the height of the zerovoltage peak and moves it close to the Fermi level of the system.

The evolution of the transmission with the bias voltage is presented in the middle and right columns of Fig. 2 for two

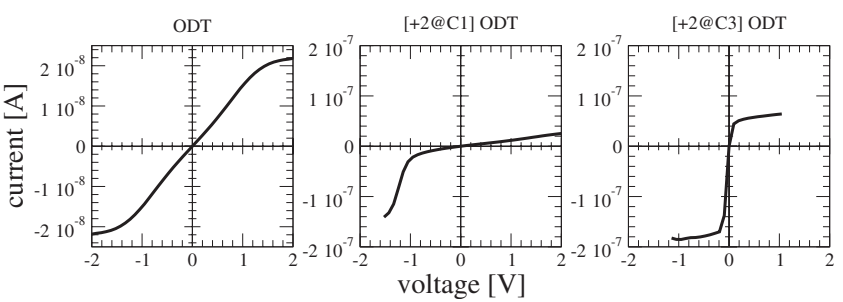

FIG. 3. $I-V$ dependence for the ODT molecular junction. Left figure: without the charge probe. Middle figure: the $+2|e|$ charge probe is near the leftmost $\mathrm{C}$ atom $(\mathrm{C} 1)$. Right figure: the $+2|e|$ charge probe is near the third $\mathrm{C}$ atom from the left (C3).

positions of the probe. For negative bias voltages (i.e., when the electrode nearest the probe charge is negative) the narrow peak moves in the direction of decreasing energy values; i.e., it approaches the Fermi level as the absolute value of the bias voltage increases. For positive bias voltages this behavior reverses. In the case of negative bias voltages the peak enters the transport window for some threshold voltage value, while it escapes the voltage window for positive biases. As shown in Fig. 3, this behavior leads to an asymmetric $I-V$ characteristic and a significant rectification ratio (note the changes in scale in the vertical axes). ${ }^{31}$ For the probe position $\mathrm{C} 3$ the rectification ratio reaches a maximum of about 7 , near the point where the sharp peak crosses the edge of the transmission window. The asymmetry of the $I-V$ characteristic and the rectification ratio both decrease when the probe is moved to the center of the junction. At the same time, the maximum current in the computed voltage range increases consistent with the increased height of the peak for position C3.

In order to understand the nature of the peak that appears in the transmission for the stronger charge probe we computed the partial density of states (PDOS) for the bonding $\mathrm{S}$ atoms when the charge probe is located near the first $\mathrm{C}$ atom from the left of the ODT chain. The results, presented in Fig. 4 , were obtained using SIESTA and mirror the transmission data of Fig. 2 obtained from SMEAGOL.

As one can see there is indeed a wide (ca. $2 \mathrm{eV}$ ) spectral feature in the PDOS on both $\mathrm{S}$ atoms. Without the charge probe the PDOSs on both $\mathrm{S}$ atoms are exactly the same, as expected for a perfectly symmetric junction. The introduction of the charge probe into the unit cell does not perturb significantly the local electronic structure of the more distant $\mathrm{S}$ atom. However, the PDOS of the $\mathrm{S}$ atom in the vicinity of

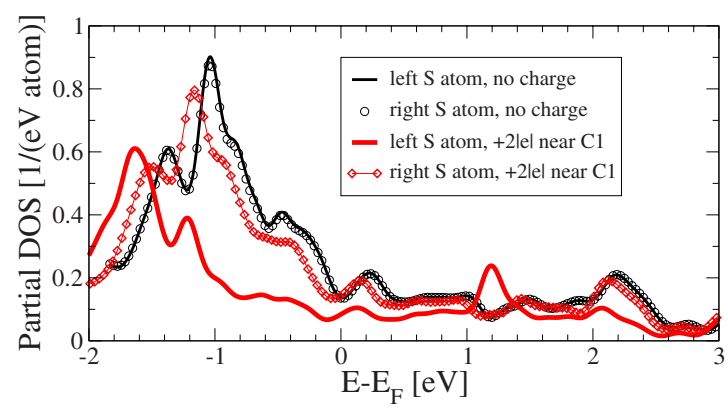

FIG. 4. (Color online) PDOS (summed over the sulfur valence orbitals) for the sulfur atoms of ODT molecule coupled to the leads at $V=0$. 
the charge probe undergoes a substantial change. First, the PDOS below $E_{\mathrm{F}}$ and especially the wide band located near $-1 \mathrm{eV}$ are considerably reduced and shifted downward. This effect is in agreement with the observed increase in the electronic charge on the $\mathrm{S}$ atom closer to the charge probe calculated from the Mulliken populations. Second, a rather narrow peak emerges near $1 \mathrm{eV}$ above $E_{\mathrm{F}}$ suggesting a close relation with the pronounced peak at the same energy in the transmission function. Since this peak is absent in the PDOS for the $\mathrm{S}$ atom in the absence of the probe and for the more distant $\mathrm{S}$ atom, we conclude that the most important effect of the charge probe is to introduce a narrow peak in the transmission function and in the PDOS of the closer S atom.

A small width of this peak suggests that it is related to the appearance of a quasilocalized level induced in the pseudogap of the ODT molecule coupled to the Au leads and generated by the strong local potential of the charge probe. The quasilocalized state, having a finite spatial extent along the molecular chain, can couple to the electronic states of both electrodes and in this way it can contribute to the transmission. If the center of the gravity of the quasilocalized state is closer to one of the electrodes, its energy dependence on the voltage will be controlled by that electrode thereby inducing an asymmetry in the $I-V$ characteristic.

To confirm the quasilocalized character of the probeinduced peak we computed the local density of states (LDOS) from the vicinity of $E \sim E_{\mathrm{F}}+1.2 \mathrm{eV}$, where the peak is found in PDOS. The results are presented in Fig. 1 for a junction without the probe (left panel) and the $+2|e|$ probe in position C1 (right panel). Without the probe LDOS is concentrated mainly near the $\mathrm{S}$ atoms and the neighboring $\mathrm{C}$ atoms with very little LDOS inside the alkane backbone. This is consistent with the approximate picture of a whole junction as a simple two-S-atom system with a strong bonding to the leads and a weak bridge between them. Including the probe creates the considerable increase in LDOS near to the probe. Note that although the biggest increase in LDOS takes place at the nearby $\mathrm{C}$ and $\mathrm{H}$ atoms, also the $\mathrm{S}$ atom closer to the probe gains noticeably more LDOS. At the same time, the more distant $\mathrm{S}$ atom is hardly influenced by the probe consistent with what we found in PDOS. On this basis we conclude that the probe-induced changes are localized near the position of the probe. Computations of LDOS for an energy window that does not include the peak in PDOS (near $E_{\mathrm{F}}+0.25 \mathrm{eV}$; not shown here) exhibit much more symmetrical LDOS more like to a system without the probe. The latter fact again confirms that the probe-induced changes are limited to a rather narrow energy window and stresses the similarity of the peak to a quasilocalized state in extended systems. $^{32}$

\section{MODEL INTERPRETATION OF THE DFT RESULTS}

To rationalize the above DFT results, we now examine a simple parametric tight-binding model of the molecule described by the following Hamiltonian:

$$
\begin{aligned}
\mathcal{H}_{M}= & t_{a} \sum_{i=1}^{N-1}\left(a_{i}^{\dagger} a_{i+1}+\text { H.c. }\right)+E_{a} \sum_{i=1}^{N} a_{i}^{\dagger} a_{i}+t_{b} \sum_{i=1}^{N-1}\left(b_{i}^{\dagger} b_{i+1}+\text { H.c. }\right) \\
& +E_{b} \sum_{i=1}^{N} b_{i}^{\dagger} b_{i}+t_{a b} \sum_{i=1}^{N-1}\left(b_{i}^{\dagger} a_{i+1}+a_{i}^{\dagger} b_{i+1}+\text { H.c. }\right) .
\end{aligned}
$$

In order to mimic the semiconducting properties of the ODT molecule it includes two electron levels per site, one corresponding to the bonding state and the other one to the antibonding state. These levels are separated by an energy gap, $E_{a}-E_{b}>0$. The operators $a, b$ are electron operators for the antibonding and the bonding levels. Note that we suppressed here the spin index, since the only effect of the spin in the absence of the magnetic field or electron repulsion reduces to the appearance of a factor of 2 in the expression for the current or the conductance. We allow for hopping between nearest-neighbor sites only. In Eq. (1), the summation goes over $N$ effective sites which can in general represent mers of an arbitrary semiconductinglike molecular chain. The coupling of the molecule to the leads will be described using energy-independent parameters $\Gamma_{a}$ and $\Gamma_{b} .{ }^{29}$ The effect of the potential ramp related to the source-drain voltage will be simulated by a site-dependent shift of the on-site energy parameters $E_{a}$ and $E_{b}$. In the same way, the effect of the local probe will be taken into account by adding a site energy shift $E_{L}$ at a site $L$. Finally, to account for the energetic inequivalence of the bonding atoms we use a site energy shift $E_{S}$ at the first and the last site of the chain. Using transfer matrices, ${ }^{33,34}$ the transmission coefficient for the above Hamiltonian can be easily computed for any finite system. The energy separation between the bands should be large enough to describe a molecular chain with semiconducting properties. When contacted to the leads the molecule will form a junction with the conductance decreasing exponentially with the increase in the chain length, as discussed in many papers (see, e.g., Ref. 35). The presence of the local shift of the potential can bring about the appearance of a localized state in the gap of the system. If the molecule is strongly coupled to the leads and the in-gap state has large enough spatial extension it can couple directly to electronic states of the leads and participate in the resonant transport through the molecule, as described in the previous section.

The orbitals of the peripheral atoms are weakly coupled by the effective hopping $t_{\text {eff }}$, which could be computed by eliminating all the other orbitals in the chain. Approximately, the whole molecule can be described in a limited energy range as a two-site system with a weak coupling $t_{\text {eff }}$ between the sites. Note that these states are located mostly on the peripheral sites and are almost symmetric to each other. The most interesting feature here is the probe-induced level, which is located close to the probe. This state can be interpreted as a localized state split off from the antibonding band by the strong attractive local potential created by the probe.

The procedure of reproducing the $a b$ initio results with the model system is facilitated by fact that Hamiltonian (1) is exactly solvable for any number of sites and parameter values and its eigenvalues are given by 

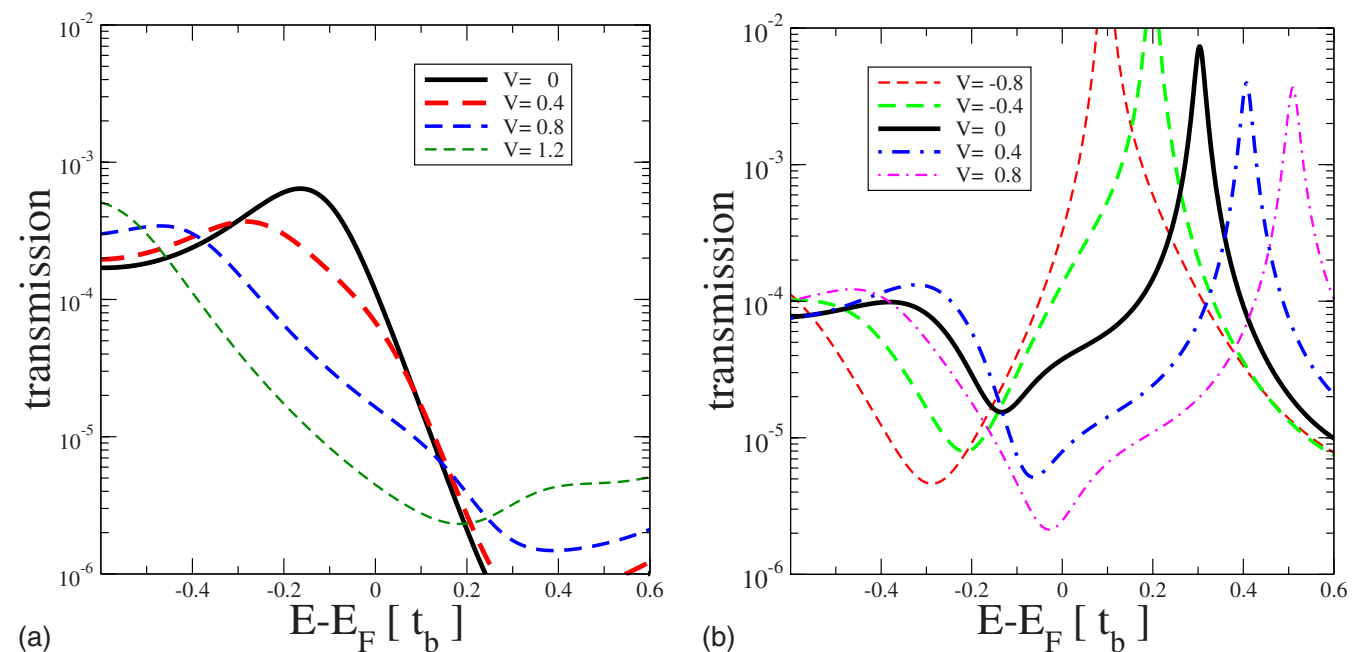

FIG. 5. (Color online) Evolution of the transmission in the vicinity of the Fermi level $\left(E_{\mathrm{F}}=0\right)$ with the bias voltage for the molecular chain of 8 mers described by the Hamiltonian $\mathcal{H}_{M}$ coupled to the leads with $\Gamma_{a, b}=0.2$. The used model parameters are as follows: $E_{a}=3$, $E_{b}=-3, t_{b}=-t_{a}=1, t_{a b}=0.11, L=2$, and $E_{S}=2.5$. Left: transmission for the system without the probe; right: transmission for the system with the probe corresponding to $E_{L}=-2.1$ located near the $L=2$ atom.

$$
\varepsilon_{\nu}^{( \pm)}=\frac{1}{2}\left[E_{a}+E_{b}+2\left(t_{a}+t_{b}\right) \cos \left(\frac{\nu \pi}{N+1}\right)\right] \pm \frac{1}{2} \sqrt{\left[E_{a}-E_{b}+2\left(t_{a}-t_{b}\right) \cos \left(\frac{\nu \pi}{N+1}\right)\right]^{2}+16 t_{a b}^{2} \cos \left(\frac{\nu \pi}{N+1}\right)^{2}}
$$

We start from setting the energy scale of our parameter unit $t_{b}$ to a value of $4 \mathrm{eV}$ which (as to the order of magnitude) is suggested by a hopping integral often used to describe the hydrocarbon systems. ${ }^{36}$ For simplicity we also put $t_{b}=-t_{a}$ and treat in what follows $t_{a b}$ as a small number. The energy gap of the spectrum of Hamiltonian (1) is given, for large $N$, by $E_{g}=\sqrt{\left(\left|E_{a}-E_{b}\right|-2\left|t_{a}-t_{b}\right|\right)^{2}+16 t_{a b}^{2}}$. On the basis of our SIESTA and SMEAGOL computations the energy gap between the main parts of the energy spectrum of the ODT molecule coupled to the leads is given by $\sim 8 \mathrm{eV}$. The last value can be well reproduced (for small $t_{a b}$ ) by taking $E_{a}=-E_{b}=3 t_{b}$. In order to fit the position of the sulfur-derived HOMO band, as well as the shape of the $2 \mathrm{eV}$ wide transmission peak just below $E_{\mathrm{F}}$ (cf. Fig. 2), we are left with three more parameters: $E_{S}, \Gamma_{a}=\Gamma_{b}$, and $t_{a b}$. By adjusting their values with a trial and error method to the $a b$ initio results we get the following: $\Gamma_{a}=\Gamma_{b}=0.2 t_{b}, E_{S}=2.5 t_{b}$, and $t_{a b}=0.11 t_{b}$. Obtained in this way the transmission function near $E_{\mathrm{F}}$ is presented in Fig. 5 (left panel). We conclude that it compares fairly well to $a b$ initio results shown in Fig. 2 (left upper panel) taking into account the very approximate nature of the two-orbital model as well as all the approximations implied by the oneparticle approach.

The plots clearly possess the same features as the transmission plots computed using DFT. In the absence of the charge probe, the transmission is dominated by a very wide peak which comes from the two levels HOMO-1 and HOMO located mainly on both ends of the chain. Since the effective hopping between the peripheral sites for this eight-site chain is much smaller than the applied intermediate values of the molecule-lead couplings, the transmission is much less than unity in the center of the peak. Suppression of the peak by the probe is due to the nonequivalent coupling of the HOMO states to the leads resulting from the asymmetric redistribution of the relevant wave functions.

In order to quantitatively simulate the effect of the external probe we shift the local energy of the bonding and antibonding levels by a common value $E_{L}=-2.1 t_{b}$ which reproduces the position of the peak from $a b$ initio computations for the $+2|e|$ probe. The height of the transmission peak is then more or less the same as the one obtained for the ODT junction (cf. Fig. 2, middle column), what independently validates our choice of $E_{L}$. At the same time the wide peak below $E_{\mathrm{F}}$ is suppressed in comparison to the case without the probe (cf. Fig. 2, lower left panel), although the value of transmission at the local maximum is by a factor of 3 lower than the one of the $a b$ initio results.

The evolution of the transmission with the bias voltage is also similar to the one observed in the DFT computation: the

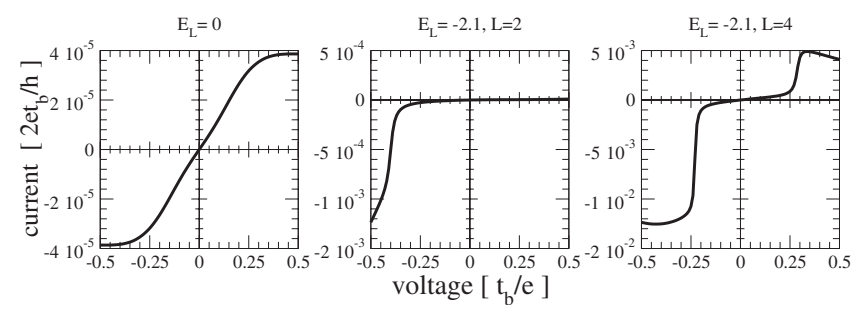

FIG. 6. $I-V$ dependence of the model system described by the Hamiltonian $\mathcal{H}_{M}$ for the same parameters as in Fig. 5. 
height of the wide peak decreases and it begins to separate into two peaks corresponding to the two orbitals, with one located mostly on the source lead and the other one mostly on the drain lead. The position of the probe-induced transmission peak corresponds rather accurately to the energy of the quasilocalized state which can be also found in PDOS (not shown here). The state couples much better to the left electrode and because of this reason its position follows rather closely the electrochemical potential of the left lead. This asymmetry of the coupling also explains the reduced value of the transmission for the peak, which is considerably smaller than unity.

The $I-V$ dependence (see Fig. 6) corresponding to the transmission data from Fig. 5 also compares well with the $a b$ initio results from Fig. 3. The current for $E_{L}=0$ shows the same smooth voltage dependence as the corresponding DFT results in the absence of the probe with an almost linear dependence in the region of the small voltage and a saturation slightly above $0.25\left|t_{b}\right| \sim 1 \mathrm{~V}$. The current for $E_{L}=-2.1 t_{b}$ and $L=2$ is also qualitatively similar to the DFT results in the presence of the probe, with a sharp rise of the absolute current for voltages near $-1 \mathrm{~V}$. In the present case, however, the rectification ratio is significantly larger than in the DFT results amounting to about 50. Another difference with the DFT results is found for the central location of the probe, where the model calculations show a gaplike shape in the current in the region $-0.25 t_{b}<e V<0.25 t_{b}$. This is due to departure of the transmission peak from $E_{F}$ at $V=0$ in the model calculation for $L=4$. We expect that some of the discrepancies can be removed by a self-consistent treatment of the model extended to include explicitly electron interactions which can modify the positions of the molecular levels with respect to the Fermi levels of the leads.

\section{CONCLUSION}

In conclusion, we have shown that the presence of a local charge in the vicinity of a molecular backbone can lead to significant rectification in the otherwise symmetric molecule.
The external local potential is shown to split a quasilocalized state from the unoccupied band. The state is centered near the probe position and significantly contributes to the electron transport when the voltage increases beyond a threshold value. The rectification is due to the strong energy dependence of the quasilocalized state on the polarization of the bias. The resulting rectification ratio depends nonlinearly on the value of the potential and the substantial $I-V$ asymmetry is observed only for strong enough potential values. We showed that the application of the external potential does not necessarily imply a current reduction; in fact we observed an increase in the current value as compared to the symmetric case when the stronger charge probe was included. The latter finding qualitatively agrees with the results of a recent experimental work. ${ }^{16}$

The proposed parametric model of the molecular junction in the field produced by the charge probe can be considered as a generic minimal model of a semiconductinglike linear molecule with an asymmetry induced by a local potential. The successful explanation of the gross features of the DFT results, with a simple choice of parameter values, suggests that the described mechanism of the rectifying behavior can be a rather common feature of such junctions. The main requirement for the device to be useful for applications is that the charge probe has to be strong enough to generate a quasilocalized level within the HOMO-LUMO energy gap, close enough to the HOMO level. In this way one can generate a quasilocalized state with a substantial spatial overlap with the electronic states of both the leads, so that it is active in transport for experimentally accessible voltage values.

\section{ACKNOWLEDGMENTS}

This work was supported by Ministry of Science and Higher Education (Poland) from sources for science in the years 2009-2012 within a research project. V.M.G.S. thanks the Spanish Ministerio de Ciencia e Innovación and the Marie Curie European ITNs FUNMOLS and NANOCTM for funding.
${ }^{1}$ A. Aviram and M. A. Ratner, Chem. Phys. Lett. 29, 277 (1974).

${ }^{2}$ A. S. Martin, J. R. Sambles, and G. J. Ashwell, Phys. Rev. Lett. 70, 218 (1993).

${ }^{3}$ J. C. Ellenbogen and J. C. Love, Proc. IEEE 88, 386 (2000).

${ }^{4}$ C. Krzeminski, C. Delerue, G. Allan, D. Vuillaume, and R. M. Metzger, Phys. Rev. B 64, 085405 (2001).

${ }^{5}$ P. E. Kornilovitch, A. M. Bratkovsky, and R. S. Williams, Phys. Rev. B 66, 245413 (2002).

${ }^{6}$ J. Taylor, M. Brandbyge, and K. Stokbro, Phys. Rev. Lett. 89, 138301 (2002).

${ }^{7}$ B. Larade and A. M. Bratkovsky, Phys. Rev. B 68, 235305 (2003).

${ }^{8}$ A. Troisi and M. A. Ratner, Nano Lett. 4, 591 (2004).

${ }^{9}$ M. Elbing, R. Ochs, M. Koentopp, M. Fischer, C. von Hanisch, F. Weigend, F. Evers, H. B. Weber, and M. Mayor, Proc. Natl. Acad. Sci. U.S.A. 102, 8815 (2005).
${ }^{10}$ R. M. Metzger, Chem. Phys. 326, 176 (2006).

${ }^{11}$ R. Liu, S. H. Ke, W. T. Yang, and H. U. Baranger, J. Chem. Phys. 124, 024718 (2006).

${ }^{12}$ H. Dalgleish and G. Kirczenow, Phys. Rev. B 73, 235436 (2006).

${ }^{13}$ N. Armstrong, R. C. Hoft, A. McDonagh, M. B. Cortie, and M. J. Ford, Nano Lett. 7, 3018 (2007).

${ }^{14}$ R. Stadler, V. Geskin, and J. Cornil, J. Phys.: Condens. Matter 20, 374105 (2008).

${ }^{15}$ M. J. Ford, R. C. Hoft, A. M. McDonagh, and M. B. Cortie, J. Phys.: Condens. Matter 20, 374106 (2008).

${ }^{16}$ I. Díez-Pérez, J. Hihath, Y. Lee, L. Yu, L. Adamska, M. A. Kozhushner, I. I. Oleynik, and N. Tao, Nat. Chem. 1, 635 (2009).

${ }^{17}$ C. Zhou, M. R. Deshpande, M. A. Reed, L. Jones II, and J. M. Tour, Appl. Phys. Lett. 71, 611 (1997). 
${ }^{18}$ M. Galperin, A. Nitzan, S. Sek, and M. Majda, J. Electroanal. Chem. 550-551, 337 (2003).

${ }^{19}$ R. L. McCreery, J. Dieringer, A. O. Solak, B. Snyder, A. M. Nowak, W. R. McGovern, and S. DuVall, J. Am. Chem. Soc. 126, 6200 (2004).

${ }^{20}$ G. J. Ashwell, A. Chwiałkowska, and L. R. H. High, J. Mater. Chem. 14, 2389 (2004).

${ }^{21}$ R. M. Metzger, J. Mater. Chem. 18, 4364 (2008).

${ }^{22}$ M. Freitag, M. Radosavljevic, Y. Zhou, A. T. Johnson, and W. F. Smith, Appl. Phys. Lett. 79, 3326 (2001).

${ }^{23}$ K.-H. Müller, Phys. Rev. B 73, 045403 (2006).

${ }^{24}$ A. R. Rocha, Víctor M. García-Suárez, S. Bailey, C. Lambert, J. Ferrer, and S. Sanvito, Phys. Rev. B 73, 085414 (2006).

${ }^{25}$ P. Ordejón, E. Artacho, and J. M. Soler, Phys. Rev. B 53, R10441 (1996); J. M. Soler, E. Artacho, J. D. Gale, A. García, J. Junquera, P. Ordejón, and D. Sánchez-Portal, J. Phys.: Condens. Matter 14, 2745 (2002).
${ }^{26}$ Reference 25 and the SIESTA manual give detailed explanations of the meaning of the simulation parameters used in our work.

${ }^{27}$ J. P. Perdew and A. Zunger, Phys. Rev. B 23, 5048 (1981).

${ }^{28}$ N. Troullier and J. L. Martins, Phys. Rev. B 43, 1993 (1991).

${ }^{29}$ T. Kostyrko, J. Phys.: Condens. Matter 14, 4393 (2002).

${ }^{30}$ L. Oroszlány, A. Kormányos, J. Koltai, J. Cserti, and C. J. Lambert, Phys. Rev. B 76, 045318 (2007).

${ }^{31}$ We were unable to obtain convergence for higher voltage ranges for some of the data presented in Figs. 2 and 3.

${ }^{32}$ H. J. Choi, J. Ihm, S. G. Louie, and M. L. Cohen, Phys. Rev. Lett. 84, 2917 (2000).

${ }^{33}$ C. Lambert, J. Phys. C 17, 2401 (1984).

${ }^{34}$ C. J. Lambert, Phys. Rev. B 29, 1091 (1984).

${ }^{35}$ C. Joachim and M. Magoga, Chem. Phys. 281, 347 (2002).

${ }^{36}$ A. P. Horsfield, P. D. Godwin, D. G. Pettifor, and A. P. Sutton, Phys. Rev. B 54, 15773 (1996). 